\title{
Enhancing Bowel Sounds by Using a Higher Order Statistics-Based Radial Basis Function Network
}

\author{
Bor-Shyh Lin, Member, IEEE, Ming-Jen Sheu, Ching-Chin Chuang, Kuan-Chih Tseng, and Jen-Yin Chen
}

\begin{abstract}
Auscultation of bowel sounds provides a noninvasive method to the diagnosis of gastrointestinal motility diseases. However, bowel sounds can be easily contaminated by background noises, and the frequency band of bowel sounds is easily overlapped with background noise. Therefore, it is difficult to enhance the noisy bowel sounds by using precise digital filters. In this study, a higher order statistics (HOS)-based radial basis function (RBF) network was proposed to enhance noisy bowel sounds. An HOS technique provides the ability of suppressing Gaussian noises and symmetrically distributed non-Gaussian noises due to their natural tolerance. Therefore, the influence of additional noises on the HOS-based learning algorithm can be reduced effectively. The simulated and experimental results show that the HOS-based RBF can exactly provide better performance for enhancing bowel sounds under stationary and nonstationary Gaussian noises. Therefore, the HOS-based RBF can be considered as a good approach for enhancing noisy bowel sounds.
\end{abstract}

Index Terms-Bowel sound, Gaussian noise, higher order statistics (HOS), radial basis function (RBF) network.

\section{INTRODUCTION}

G ASTROINTESTINAL motility is closely related to gastrointestinal problems, and the auscultation of bowel sounds is usually used for the diagnosis in internal medicine and postsurgery. However, the auscultation of bowel sounds requires clinical experiences of physicians and is easily affected by environmental noise [1]. Therefore, the quantitative and qualitative evaluations of bowel sounds for gastrointestinal diseases still encounter some difficulties.

Manuscript received July 12, 2012; revised December 17, 2012; accepted January 25, 2013. Date of publication February 1, 2013; date of current version May 1, 2013. This work was supported by the National Science Council of Taiwan under Grant NSC 100-2221-E-009-007 and Grant NSC 100-2622-E009-012-CC3.

B.-S. Lin is with the Institute of Imaging and Biomedical Photonics and Biomedical Electronics Translational Research Center, National Chiao Tung University, Hsinchu 300, Taiwan, and also with the Department of Medical Research, Chi Mei Medical Center, Tainan 710, Taiwan (e-mail: borshyhlin@ gmail.com).

M.-J. Sheu is with the Division of Gastroenterology and Hepatology, Chi Mei Medical Center, Tainan 710, Taiwan, and also with the Department of Medicinal Chemistry, Chia Nan University of Pharmacy and Science, Tainan 717, Taiwan.

C.-C. Chuang is with the Institute of Imaging and Biomedical Photonics, National Chiao Tung University, Hsinchu 300, Taiwan.

K.-C. Tseng is with the Division of Gastroenterology and Hepatology, Chi Mei Medical Center, Tainan 710, Taiwan.

J.-Y. Chen is with the Department of Anesthesiology, Chi Mei Medical Center, Tainan 710, Taiwan, and also with the Department of the Senior Citizen Service Management, Chia Nan University of Pharmacy and Science, Tainan 717, Taiwan.

Digital Object Identifier 10.1109/JBHI.2013.2244097
The frequency distribution of bowel sounds is between about 100 and $800 \mathrm{~Hz}$ and is easily overlapped with environmental noise. Thus, it is difficult to enhance noisy bowel sounds by using precise digital filters. Several studies utilized the adaptive filtering technique to enhance noisy bowel sounds. In 1997, Mansy and Sandler used an adaptive noise canceller with the Widrow-Hoff least-mean-square (LMS) algorithm to reduce the distortion of gastrointestinal acoustic phenomena due to heart sounds [2]. Abdominal sounds are usually nonlinear, and thus, a great number of filter taps are usually required to effectively enhance nonlinear signals. In order to effectively enhance nonlinear signals, using neural network filters [3]-[5], which can well approximate any continuous nonlinear function with a small amount of training data and much fewer filter taps, may be a good alternative. In 1997, Gandhi and Ramamurti used a threelayer neural network to detect non-Gaussian noise [3]. However, the learning of multilayer perceptron (MLP) network is slow and global, and the estimate of MLP network easily traps at a local minimum during the learning procedure. In 1995, Billings and Fung used a recurrent radial basis function (RBF) network to cancel noise [4]. Cha and Kassam used the RBF network to eliminate interference [5]. However, additional noise usually affects the adaptation of weights in the RBF network. Therefore, reducing the influence of additional noise on the performance of enhancing bowel sounds for RBF network is important.

Recently, a higher order statistics (HOS) technique has been developed and applied for signal enhancement [6]-[13]. HOS techniques provide the ability of suppressing Gaussian noise and symmetrically distributed non-Gaussian noise due to their natural tolerance [14], [15]. In this study, the HOS-based RBF network was proposed to enhance noisy bowel sounds. Bowel sounds usually contain high non-Gaussianity due to the feature of explosive sounds [16]. By using HOS techniques, the non-Gaussian property of bowel sounds can be enhanced effectively. Here, an adaptive line enhancement (ALE) scheme, which uses the delayed input signal as the reference signal, is utilized, and only single channel of bowel sounds is required for signal enhancement. The performance of enhancing noisy bowel sounds under different noise state was investigated, and finally, the HOS-based RBF was tested and validated at Chimei Medical Center. This paper is organized as follows. The basic schemes of RBF network and HOS-based RBF for signal enhancement are introduced in Section II. The performance of enhancing bowel sounds using different methods is investigated in Section III. In Section IV, conclusions are drawn. 


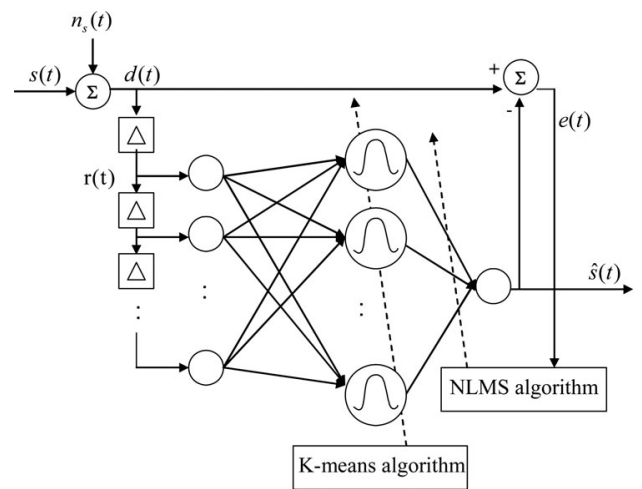

Fig. 1. Basic scheme of RBF network for signal enhancement.

\section{METHODS}

\section{A. RBF Network for Signal Enhancement}

RBF was first derived by Broomhead and Lowe in 1988 [17]. It is a special three-layer network containing an input layer, a hidden layer, and an output layer. The hidden layer performs a nonlinear transformation role which maps the input space onto the output space. Therefore, by using RBF network, the surface in a multidimensional space, which provides a best fit to training data, can be found out [18], [19].

The basic scheme of RBF network for signal enhancement is shown in Fig. 1. Let the desired signal $d(t)$ be

$$
d(t)=s(t)+n_{s}(t)
$$

where $s(t)$ and $n_{s}(t)$ denote the noise-free bowel sound and additional noise at the iteration $t$, respectively. Based on the scheme of ALE [20], the delayed version of $d(t)$ can be used as the reference signal $r(t)$ and is given by

$$
r(t)=d(t-\Delta) \text {. }
$$

Here, the prediction depth $\Delta$ is usually selected by the unit of sampling period. Let $N_{0}$ and $N_{1}$ denote the number of the input and hidden nodes, respectively; the estimate $\hat{s}(t)$ of $s(t)$ can be calculated by

$$
\hat{s}(t)=\boldsymbol{\delta}(t)^{T} \mathbf{w}(t)
$$

where $\boldsymbol{\delta}(t)=\left[\delta_{1}(t), \delta_{2}(t), \ldots, \delta_{N_{1}}(t)\right]^{T}$ denotes the output vector of the hidden nodes, generated by Gaussian basis function, and $\mathbf{w}(t)=\left[w_{1}(t), w_{2}(t), \ldots, w_{N_{1}}(t)\right]^{T}$ denotes the weight vector connecting the hidden nodes with the output node. The output of Gaussian basis function in the $k$ th hidden node can be defined by

$$
\delta_{k}(t)=\exp \left(-\frac{\left\|\mathbf{r}(t)-\mathbf{v}_{\mathbf{k}}(t)\right\|^{2}}{2 \rho^{2}(t)}\right)
$$

where $\|\cdot\|$ denotes the Euclidean norm, $\mathbf{r}(t)=[r(t)$, $\left.r(t-1), \ldots, r\left(t-N_{0}+1\right)\right]^{T}$ is the vector of the input nodes, and $\mathbf{v}_{\mathbf{k}}(t)$ denotes the center in the $k$ th hidden node. In addition, $\rho(t)$ denotes the width of the centers and can be defined as the variance of the input vector. The center $\mathbf{v}_{\mathbf{k}}(t)$ can be obtained by the k-means clustering algorithm [21], which is a self-organized learning procedure, and the weight vector $\mathbf{w}(t)$

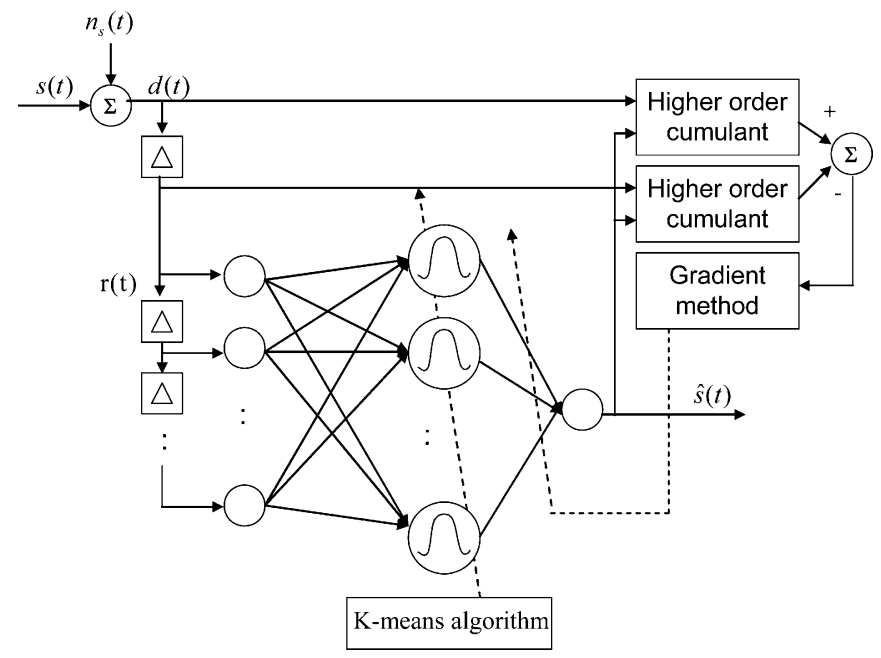

Fig. 2. Basic scheme of higher order statistics-based RBF network for signal enhancement.

is commonly adapted by the normalized LMS algorithm [20]. The weight vector can be adapted by

$$
\mathbf{w}(t+1)=\mathbf{w}(t)+\frac{\frac{\mu_{w}}{\left(1+\boldsymbol{\delta}^{T}(t) \boldsymbol{\delta}(t)\right)}}{\boldsymbol{\delta}}(t)(d(t)-\hat{s}(t))
$$

where $\mu_{w}$ denotes the learning rate. Obviously, adapting weights is easily affected by additional noise $n_{s}(t)$ due to that $e(t)$ is correlated with additional noise $n_{s}(t)$, i.e., $e(t)=d(t)-\hat{s}(t)=$ $s(t)+n_{s}(t)-\hat{s}(t)$.

\section{B. HOS-Based RBF Network for Signal Enhancement}

The basic scheme of HOS-based RBF network for signal enhancement is shown in Fig. 2. For a set of real stationary variables $\left\{z_{i}(t)\right\}, \quad i=1,2,3, \ldots$, the $n$ th-order cross cumulant of $\left\{z_{i}(t)\right\}$ can be defined as follows:

$$
\begin{gathered}
C_{z_{1} z_{2} \ldots z_{n}}\left(\tau_{1}, \tau_{2}, \ldots, \tau_{n-1}\right) \equiv \operatorname{Cum}\left[z_{1}(t), z_{2}\left(t+\tau_{1}\right),\right. \\
\left.z_{3}\left(t+\tau_{2}\right), \ldots, z_{n}\left(t+\tau_{n-1}\right)\right] .
\end{gathered}
$$

Here, Cum [.] denotes the cumulant operator [14]. Under the assumption that the $n$ th-order cumulants $(n>2)$ of the desired and reference signals exist, they can be represented by

$$
\begin{aligned}
& C_{d r r \ldots r}\left(\tau_{1}, \tau_{2}, \ldots, \tau_{n-1}\right) \\
& =\operatorname{Cum}\left[d(t), r\left(t+\tau_{1}\right), r\left(t+\tau_{2}\right), \ldots, r\left(t+\tau_{n-1}\right)\right] .
\end{aligned}
$$

Since $s(t)$ and $n_{s}(t)$ in the desired and reference signal are independent, and the $n$th cumulant of Gaussian noises is identically zero, (7) can then be simplified as

$$
\begin{array}{r}
C_{d r \ldots r}\left(\tau_{1}, \tau_{2}, \ldots, \tau_{n-1}\right)=\operatorname{Cum}\left[s(t), s\left(t+\tau_{1}-\Delta\right), \ldots,\right. \\
\left.s\left(t+\tau_{n-1}-\Delta\right)\right]
\end{array}
$$$$
=C_{s s \ldots s}\left(\tau_{1}-\Delta, \tau_{2}-\Delta, \ldots, \tau_{n-1}-\Delta\right) .
$$

Similarly, the $n$th cumulant of the filtered output signal can also be represented by

$C_{\hat{s} r \ldots . r}\left(\tau_{1}, \tau_{2}, \ldots, \tau_{n-1}\right)=C_{\hat{s} s s \ldots s}\left(\tau_{1}-\Delta, \tau_{2}-\Delta, \ldots, \tau_{n-1}-\Delta\right)$. 
From (8) and (9), it is obvious that the influence of additional noise $n_{s}(t)$ on the learning algorithm of HOS-based RBF can be reduced effectively, due to that the difference between the higher order cumulants of $d(t)$ and the filtered output $\hat{s}(t)$ are used as the learning criterion $\xi$. And the learning criterion $\xi$ can then be expressed by

$$
\begin{aligned}
\xi & =\sum_{\tau_{1}} \sum_{\tau_{2}} \ldots \sum_{\tau_{n-1}} \frac{1}{2}\left[C_{\hat{s} r r \ldots r}\left(\tau_{1}, \tau_{2}, \ldots, \tau_{n-1}\right)\right. \\
& \left.-C_{d r r \ldots r}\left(\tau_{1}, \tau_{2}, \ldots, \tau_{n-1}\right)\right]^{2} \\
& =\sum_{\left(\tau_{1}, \tau_{2}, \ldots, \tau_{n-1}\right) \in \boldsymbol{\Gamma}} \sum_{\left.\left.\tau_{n-1}-\Delta\right)-C_{s s \ldots s}\left(\tau_{1}-\Delta, \tau_{2}-\Delta, \ldots, \tau_{n-1}-\Delta\right)\right]^{2}}\left[C _ { \hat { s } s s \ldots s } \left(\tau_{1}-\Delta, \tau_{2}-\Delta, \ldots,\right.\right. \\
& =\sum \sum_{\left(\tau_{1}, \tau_{2}, \ldots, \tau_{n-1}\right) \in \boldsymbol{\Gamma}} \quad \sum \frac{1}{2}\left[\sum_{j=1}^{N_{1}} w_{j} C_{\delta_{j} r r \ldots r}\left(\tau_{1}, \tau_{2}, \ldots, \tau_{n-1}\right)\right. \\
& \left.-C_{d r r \ldots r}\left(\tau_{1}, \tau_{2}, \ldots, \tau_{n-1}\right)\right]^{2} .
\end{aligned}
$$

Equation (10) can be rewritten in a matrix form

$$
\xi=\frac{1}{2}\left[\mathbf{C}_{\delta \mathrm{rr} \ldots \mathrm{r}} \mathbf{w}-\mathbf{C}_{d \mathrm{rr} \ldots \mathrm{r}}\right]^{2}
$$

Here, $\mathbf{C}_{\delta \mathrm{rr} \ldots \mathrm{r}}$ and $\mathbf{C}_{d \mathrm{rr} \ldots \mathrm{r}}$ are an $N_{\Gamma} \times N_{1}$ matrix and an $N_{\Gamma} \times 1$ column vector, and $N_{\Gamma}$ denotes the number of points in the set $\Gamma$. In order to minimize $\xi$, the gradient-descent method was used. The gradient of $\xi$ is given by

$$
\nabla \mathbf{w}(t) \equiv \frac{\partial \xi}{\partial \mathbf{w}(t)}=-\mathbf{C}_{\delta \mathrm{rr} \ldots \mathrm{r}}^{T}(t)\left[\mathbf{C}_{d \mathrm{rr} \ldots \mathrm{r}}(t)-\mathbf{C}_{\delta \mathrm{rr} \ldots \mathrm{r}}(t) \mathbf{w}(t)\right]
$$

Consequently, the adaptation formula can be given by

$$
\mathbf{w}(t+1)=\mathbf{w}(t)-\frac{\mu_{w}}{\left(1+\operatorname{tr}\left(\mathbf{C}_{\boldsymbol{\delta} \mathrm{rr} \ldots \mathrm{r}}^{T} \mathbf{C}_{\boldsymbol{\delta} \mathrm{rr} \ldots \mathrm{r}}\right)\right)} \nabla \mathbf{w}(t) .
$$

Under the assumption that $s(t)$ approximates to $\hat{s}(t)$, the learning criterion in (10) will be close to optimal. Therefore, by using the HOS-based learning algorithm, the influence of additional noises $n_{s}(t)$ on $\mathbf{C}_{d \mathrm{rr} . . . \mathrm{r}}$ can be eliminated and provide better performance. In this paper, the HOS-based RBF with the third-order statistics was used for enhancing bowel sounds. For a zero-mean signal, the estimate of the third-order cumulant can be recursively computed by

$$
\hat{C}_{z_{1} z_{2} z_{3}}\left(t ; \tau_{1}, \tau_{2}\right)=<z_{1}(t) z_{2}\left(t+\tau_{1}\right) z_{3}\left(t+\tau_{2}\right)>.
$$

Here, the operation $<\cdot>$ is defined as follows:

$$
<f(t)>=\lambda<f(t-1)>+(1-\lambda) f(t)
$$

where $\lambda$ is a forgetting factor.

\section{RESUltS AND DISCUSSIONS}

\section{A. Performance Comparison for Enhancing Bowel Sounds Under Stationary Gaussian Noise}

In this section, the performances of enhancing bowel sounds under stationary Gaussian noise by using different methods,
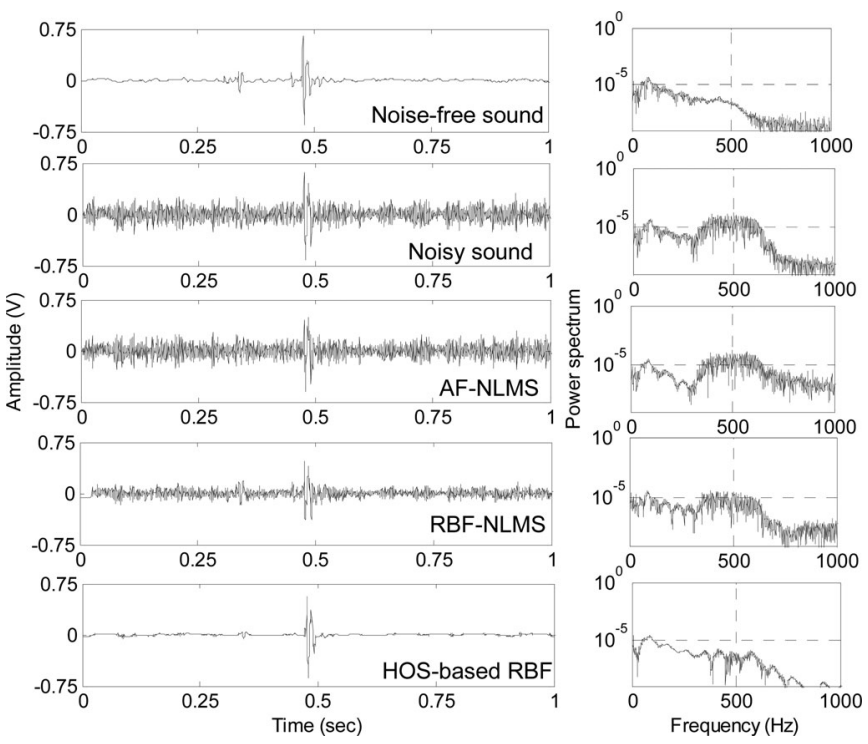

Fig. 3. Noise-free bowel sound, simulated noisy bowel sound with an SNR of $-6 \mathrm{~dB}$, and filtered bowel sounds by using different methods.

which include adaptive filter with normalized LMS algorithm (AF-NLMS), adaptive RBF filter with normalized LMS algorithm (RBF-NLMS), and the HOS-based RBF, were investigated. In this study, ten trials of 1-min bowel sounds (five healthy control cases and five adhesive ileus cases) recorded in the anechoic chamber were used to validate the performance of the proposed methods. The frequency of peristalsis is about $3-15$ events/min, and the length of the bowel sound is about $1-3 \mathrm{~s}$. Therefore, in order to clearly present the effect of different methods on the enhancement of bowel sounds, one of the peristalsis events from adhesive ileus cases was selected as the pattern of noise-free bowel sounds in this study. The simulated noisy bowel sounds with different signal-to-noise ratio (SNR) were generated for simulations. Here, the definition of SNR is given by

$$
\mathrm{SNR}=20 \log _{10}\left(\frac{A_{s}}{A_{n}}\right)
$$

where $A_{s}$ and $A_{n}$ denote the root mean square of noise-free bowel sound and noise, respectively. Fig. 3 shows the noisefree bowel sound, noisy bowel sound with an SNR of $-6 \mathrm{~dB}$, and the results of filtered bowel sounds by using 32 filter-order AF-NLMS $\left(\mu_{w}=0.8\right)$, RBF-NLMS $\left(N_{0}=32, N_{1}=32\right.$, and $\left.\mu_{w}=0.8\right)$, and HOS-based RBF $\left(N_{0}=32, N_{1}=32, \mu_{w}=0.8\right.$, and $\lambda=0.1$ ), respectively. It shows that the SNR of the filtered bowel sound by using AF-NLMS, RBF-NLMS, and HOS-based $\mathrm{RBF}$ are about $-0.18,4.76$, and $12.18 \mathrm{~dB}$, respectively.

Next, the effect of selecting the learning rate $\mu_{\omega}$ on the performance was investigated. Fig. 4 shows the comparison between the performances of enhancing bowel sounds corresponding to different learning rate. Obviously, the HOS-based RBF with $\mu_{\omega}=1.6$ can provide better performance for enhancing bowel sounds. Therefore, by using the HOS technique, the influence of additional noise on adapting weights can be reduced effectively. 


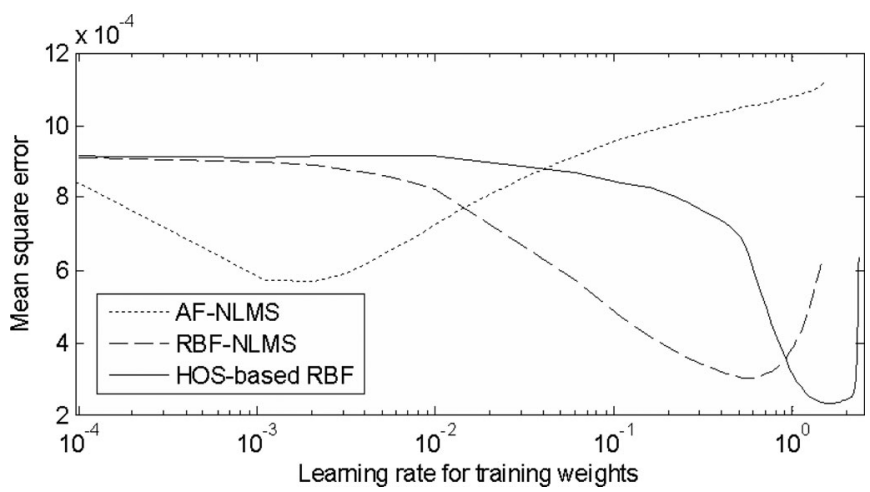

Fig. 4. Performance comparison for enhancing noisy bowel sounds corresponding to different learning rates by using different methods.

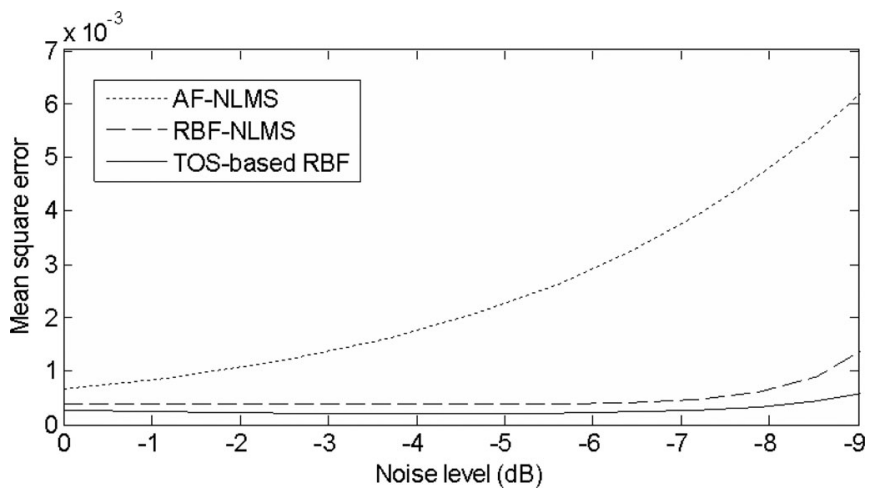

Fig. 5. Performance comparison of enhancing noisy bowel sounds by using different methods under different noise levels.

Next, the performances of enhancing noisy bowel sounds under different noise levels were investigated. Here, the simulated noisy bowel sounds with the SNR of $-9-0 \mathrm{~dB}$ were generated for simulations. Fig. 5 shows the results for the performance of enhancing noisy bowel sounds under different noise level. Both HOS-based RBF and RBF-NLMS can provide better performance under different noise level. Obviously, the performance of enhancing bowel sounds for AF-NLMS rapidly becomes poorer when the SNR of noisy bowel sounds becomes poorer. However, the performance of enhancing bowel sounds for HOS-based RBF is most insensitive to the noise level.

\section{B. Performance Comparison for Enhancing Bowel Sounds Under Nonstationary Gaussian Noise}

The performance of enhancing noisy bowel sounds under nonstationary Gaussian noise was investigated in this section. Here, four types of Gaussian noise, as shown in Fig. 6(a), were used to generate nonstationary noise. Here, the type of additional Gaussian noises was randomly selected and its magnitude was also varied every $25 \mathrm{~ms}$ to generate nonstationary Gaussian noises with the SNR of $15-0$ dB. Fig. 6(b) shows the results of filtered bowel sounds by using 32 filter-order AF-NLMS ( $\mu_{w}=$ $0.8)$, RBF-NLMS ( $N_{0}=32, N_{1}=32$, and $\left.\mu_{w}=0.8\right)$, and HOS-based RBF $\left(N_{0}=32, N_{1}=32, \mu_{w}=0.8\right.$, and $\left.\lambda=0.1\right)$. The simulated result shows that AF-NLMS and RBF-NLMS are relatively sensitive to the SNR variation of additional noise, but (a)
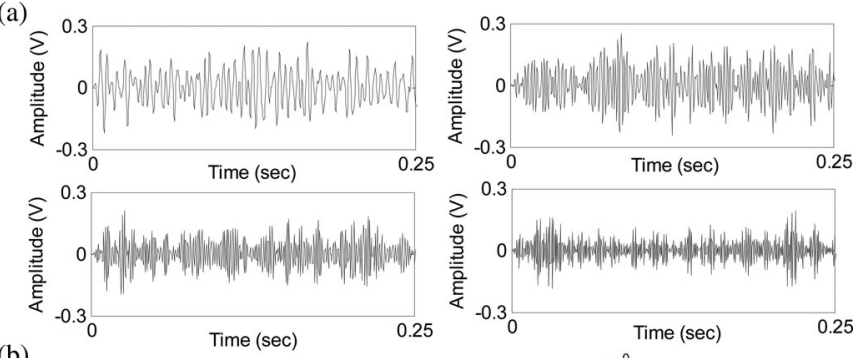

(b)
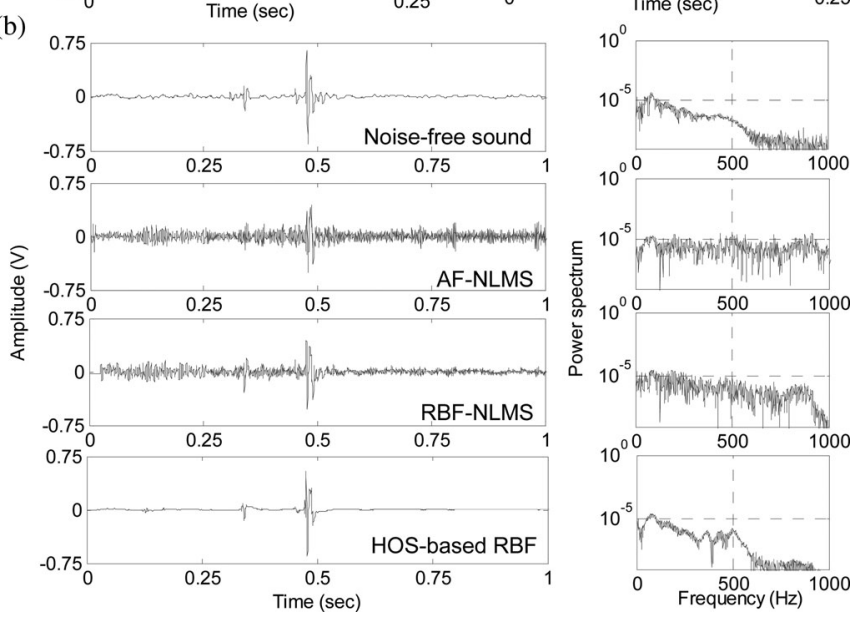

Fig. 6. (a) Four types of Gaussian noises used to generate nonstationary noise, and (b) filtered bowel sounds by using different methods.

the influence of the SNR variation of additional noise for HOSbased RBF is negligible. Obviously, the efficiency of HOS-based RBF for enhancing noisy bowel sounds under nonstationary Gaussian noise is most significant.

Next, the performance of enhancing noisy bowel sounds under environment noise for different methods was also investigated. Two types of crowd-indoor sounds from the Sound Ideas [22], as shown in Fig. 7(a), were used to simulate environment noise in the Emergency room or the Intensive Care Unit. Fig. 7(b) and (c) shows the results obtained by AF-NLMS, RBFNLMS, and HOS-based RBF for two types of crowd-indoor sounds, respectively. In this simulation, the SNR of noisy bowel sounds was set to $-3 \mathrm{~dB}$. For case I noise, the SNRs of filtered noisy bowel sound by using AF-NLMS, RBF-NLMS, and HOSbased RBF are about 4.74, 6.23, and $8.55 \mathrm{~dB}$, respectively. For case II noise, the SNRs of filtered noisy bowel sound by using AF-NLMS, RBF-NLMS, and HOS-based RBF are 5.2, 6.27, and $9.02 \mathrm{~dB}$, respectively. From the above simulated results, the HOS-based RBF presented the better performance for enhancing noisy bowel sounds under different environment noise.

\section{Performance Evaluation for Enhancing Real Bowel Sounds}

In this section, the performance of enhancing real bowel sounds was evaluated by questionnaire test. Twenty trials of bowel sounds with environmental noise (ten healthy control cases and ten adhesive ileus cases) recorded in the common ward were used for test. Here, healthy controls are from health management center. The patient group is from hospital inpatients. They were all in fasting status at least for $6 \mathrm{~h}$. Each bowel sound trial was processed by using AF-NLMS, 


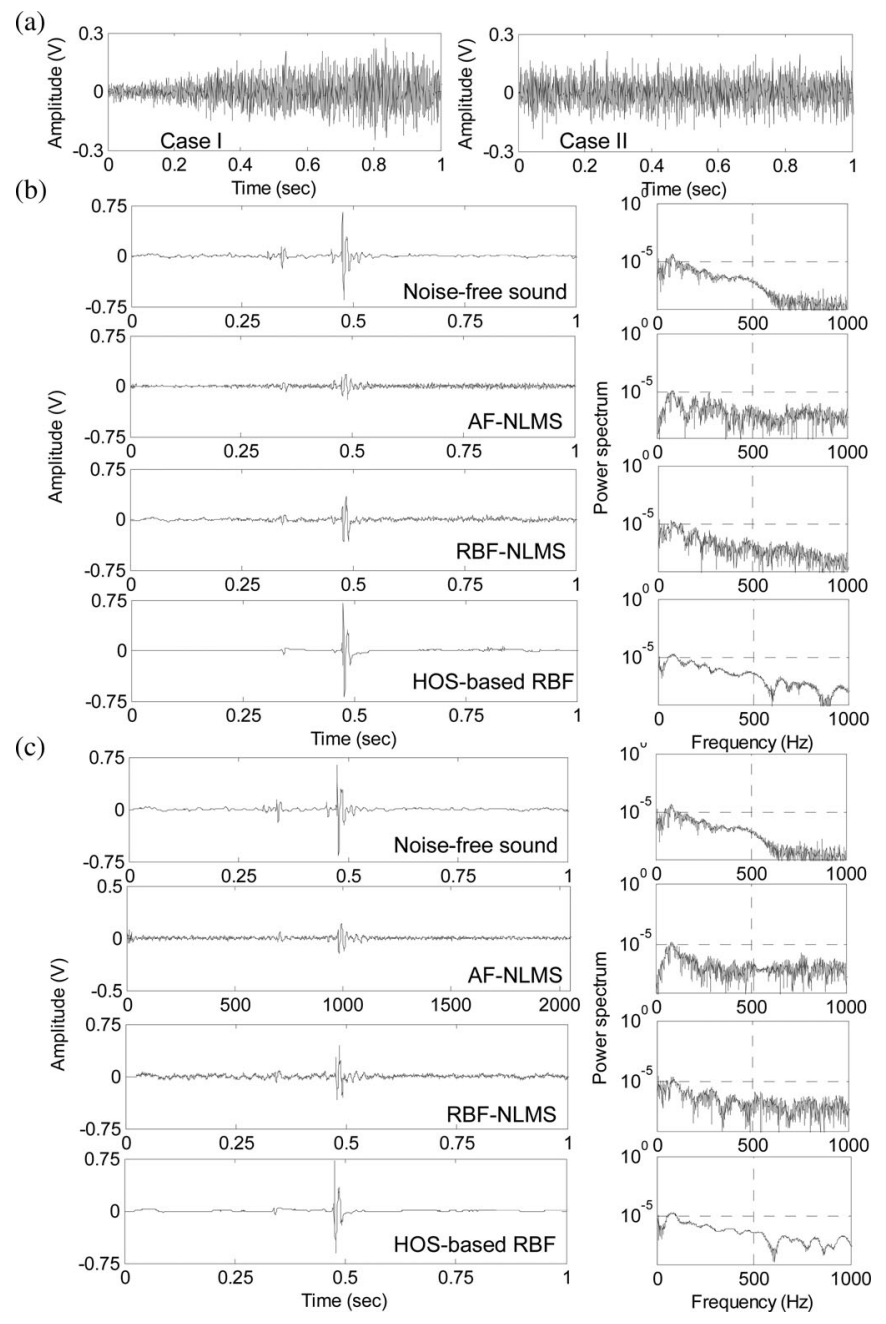

Fig. 7. (a) Two types of crowd-indoor sounds: case I and case II, (b) filtered bowel sounds for case I noise, and (c) filtered bowel sounds for case II noise.

RBF-NLMS, and HOS-based RBF, respectively. For each trial, the results of bowel sound processed by different methods were randomly sorted to avoid the physician recognizing the processing methods. A total of 12 physicians from the Division of Gastroenterology and Hepatology, Chimei Medical Center, Taiwan, who own extensive experience in the auscultation diagnosis of bowel sounds, were invited to attend this questionnaire test. The physicians were instructed to hear the original bowel sound first, and then hear these filtered bowel sounds in sequence to compare the efficiency of these unknown methods on enhancing the original bowel sounds. Here, the efficiency of enhancing bowel sounds will be rated in the level range between 1 and 5. Finally, MATLAB was used to perform analysis of variance (ANOVA) analysis of the questionnaire exam.

Fig. 8 shows the questionnaire results for the efficiency level of enhancing bowel sounds. Here, one-way ANOVA was used for analysis, and the sign $* *$ denotes that the efficiency difference is significant $(p<0.01)$. The mean and standard deviation for the level of enhancing effect by using AF-NLMS, RBFNLMS, and HOS-based RBF are $1.08 \pm 0.33,3.51 \pm 0.83$, and $4.62 \pm 0.76$, respectively. Obviously, the performance of

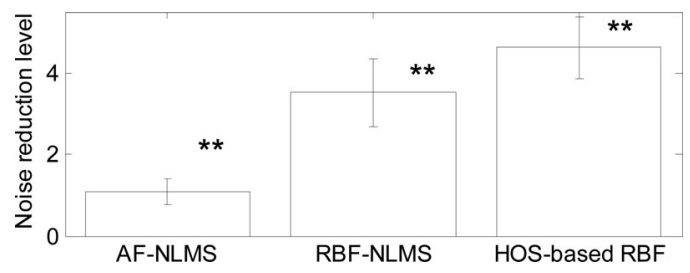

Fig. 8. Results of questionnaire examination for enhancing noisy bowel sounds.

enhancing noisy bowel sound by using HOS-based RBF is best. RBF-NLMS can also provide a good performance for enhancing bowel sounds. However, the computational complexity of RBF-NLMS is significantly smaller than that of HOS-based RBF.

\section{CONCLUSION}

The HOS-based RBF network was proposed for enhancing noisy bowel sounds in this study. Based on the nature tolerance of HOS on suppressing Gaussian noises and symmetrically distributed non-Gaussian noises, the learning criterion designed by using higher order cumulants can reduce the influence of additional noise on adapting weights effectively. Simulated results validated that using the HOS-based RBF can effectively enhance bowel sounds under both stationary and nonstationary Gaussian noises. Finally, the efficiency of enhancing real bowel sounds for different methods was also evaluated by questionnaire examination. From the questionnaire result, it shows that both HOS-based RBF and RBF-NLMS can provide good performances for enhancing real bowel sounds. Although the performance of HOS-based RBF is better than that of RBF-NLMS, the computational complexity of RBF-NLMS is far less than that of HOS-based RBF. Therefore, both HOS-based RBF and RBF-NLMS can be a preferred solution for enhancing bowel sounds.

\section{REFERENCES}

[1] B. L. Craine, M. L. Silpa, and C. J. O'Toole, "Two-dimensional positional mapping of gastrointestinal sounds in control and functional bowel syndrome patients," Digestive Diseases Sci., vol. 47, pp. 1290-1296, 2002.

[2] H. A. Mansy and R. H. Sandler, "Bowel sound signal enhancement using adaptive filtering - Separating heart sounds from gastrointestinal acoustic phenomena," IEEE Eng. Med. Biol. Mag., vol. 16, no. 6, pp. 105-117, Nov./Dec. 1997.

[3] P. P. Gandhi and V. Ramamurti, "Neural networks for signal detection in non-Gaussian noise," IEEE Trans. Signal Process., vol. 45, no. 11, pp. 2846-2851, Nov. 1997.

[4] S. A. Billings and C. F. Fung, "Recurrent radial basis function networks for adaptive noise cancellation," Neural Netw., vol. 8, pp. 273-290, 1995.

[5] I. Cha and S. A. Kassam, "Interference cancellation using radial basis function networks," Signal Process., vol. 47, pp. 247-268, 1995.

[6] B. M. Sadler, G. B. Giannakis, and K.-S. Li, "Estimation and detection in non-Gaussian noise using higher order statistics," IEEE Trans. Signal Process., vol. 42, no. 10, pp. 2729-2741, Oct. 1994.

[7] L. J. Hadjileontiadis and S. M. Panas, "Adaptive reduction of heart sounds from lung sounds using fourth-order statistics," IEEE Trans. Biomed. Eng., vol. 44, no. 7, pp. 642-648, Jul. 1997.

[8] H. M. Ibrahim, R. R. Gharieb, and M. M. Hassan, "A higher order statistics-based adaptive algorithm for line enhancement," IEEE Trans. Signal Process., vol. 47, no. 2, pp. 527-532, Feb. 1999. 
[9] K. Li, M. N. S. Swamy, and M. O. Ahmad, "An improved voice activity detection using higher order statistics," IEEE Trans. Speech Audio Process., vol. 13, no. 5, pp. 965-974, Sep. 2005.

[10] B. S. Lin, B. S. Lin, F. C. Chong, and F. P. Lai, "A functional link network with higher order statistics for signal enhancement," IEEE Trans. Signal Process., vol. 54, no. 12, pp. 4821-4826, Dec. 2006.

[11] M. E. Hassouni, H. Cherifi, and D. Aboutajdine, "HOS-based image sequence noise removal," IEEE Trans. Image Process., vol. 15, no. 3, pp. 572-581, Mar. 2006.

[12] B. S. Lin, B. S. Lin, F. C. Chong, and F. P. Lai, "Higher-order-statisticsbased radial basis function networks for signal enhancement," IEEE Trans. Neural Netw., vol. 18, no. 3, pp. 823-832, May 2007.

[13] B. S. Lin, B. S. Lin, F. C. Chong, and F. P. Lai, "Higher order statisticsbased radial basis function network for evoked potentials," IEEE Trans. Biomed. Eng., vol. 56, no. 1, pp. 93-100, Jan. 2009.

[14] C. L. Nikias and A. P. Petropulu, Higher-Order Spectra Analysis: A Nonlinear Signal Processing Framework. Englewood Cliffs, NJ, USA Prentice-Hall, 1993.

[15] C. L. Nikias and J. M. Mendel, "Signal processing with higher-order spectra," IEEE Signal Process. Mag., vol. 10, no. 3, pp. 10-37, Jul. 1993.

[16] I. T. Rekanos and L. J. Hadjileontiadis, "An iterative kurtosis-based technique for the detection of nonstationary bioacoustic signals," Signal Process., vol. 86, pp. 3787-3795, 2006.

[17] D. S. Broomhead and D. Lowe, "Multivariable functional interpolation and adaptive networks," Complex Syst., vol. 2, pp. 321-355, 1988.

[18] J. Park and I. W. Sandberg, "Universal approximation using radial basis function networks," Neural Comput., vol. 3, pp. 246-257, 1991.

[19] J. Park and I. W. Sandberg, "Approximation and radial basis function networks," Neural Comput., vol. 5, pp. 305-316, 1993.

[20] S. Haykin, Adaptive Filter Theory, 2nd ed. Englewood Cliffs, NJ, USA: Prentice-Hall, 1991.

[21] J. C. Bezdek, Pattern Recognition With Fuzzy Objective Function Algorithms. New York, USA: Plenum, 1981.

[22] Sound Ideas, XV Sound Effects Series, (2001). [Online]. Available: http://www.sound-ideas.com/xv.html

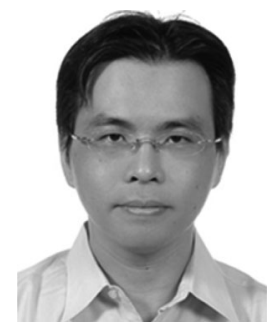

Bor-Shyh Lin (M'02) received the B.S. degree from National Chiao Tung University, Hsinchu, Taiwan, in 1997, and the M.S. and Ph.D. degrees both in electrical engineering from National Taiwan University, Taipei, Taiwan, in 1999 and 2006, respectively.

$\mathrm{He}$ is currently an Assistant Professor at the Institute of Imaging and Biomedical Photonics, National Chiao Tung University, Hsinchu. His research interests include biomedical circuits and systems, biomedical signal processing, and biosensor.

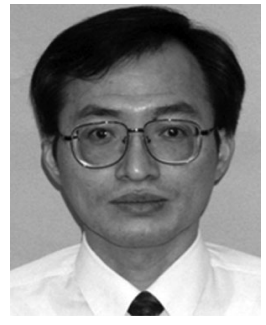

Ming-Jen Sheu received the B.M. degree from Chung Shan Medical College, Taichung, Taiwan, in 1986.

He is currently the Director of the Division of Gastroenterology, Department of Internal Medicine, Chi Mei Medical Center, Tainan, Taiwan. His research interests include gastroenterology, hepatology, and internal medicine.

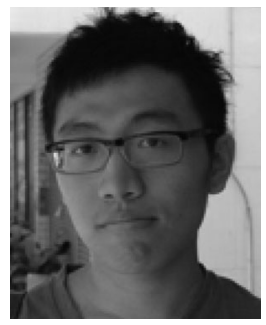

Ching-Chin Chuang received the B.S. degree from National Central University, Taoyuan, Taiwan, in 2010, and the M.S. degree from the Institute of Imaging and Biomedical Photonics, National Chiao Tung University, Hsinchu, Taiwan, in 2012.

His research interests include biomedical circuits and systems, and biomedical signal processing.

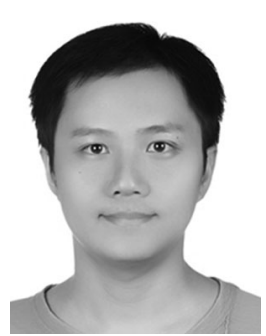

Kuan-Chih Tseng received the B.M. degree from Chung Shan Medical University, Taichung, Taiwan, in 2006.

$\mathrm{He}$ is currently a Fellow with the Division of Gastroentero-hepatology, Department of Internal Medicine, Chi Mei Medical Center, Tainan, Taiwan. His research interests are in the areas of gastroenterology and internal medicine.

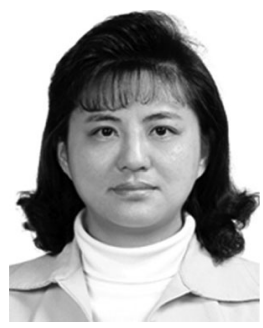

Jen-Yin Chen received the M.D. degree from the School of Medicine, China Medical University, Taichung, Taiwan, in 1986, and the Master degree in food science and applied biotechnology from National Chung Hsing University, Taichung, in 2004.

She is currently an Assistant Professor in the Department of the Senior Citizen Service Management, Chia Nan University of Pharmacy and Science, Tainan, Taiwan. Her research interests include clinical medicine, biostatistics, and nutrition. 